\title{
Evaluation of functional outcome of bilateral kidney tumors after sequential surgery
}

Jung Kwon Kim ${ }^{1 \dagger}$, Hwanik Kim ${ }^{1 \dagger}$, Hakmin Lee ${ }^{1}$, Jong Jin Oh ${ }^{1,2}$, Sangchul Lee ${ }^{1}$, Sung Kyu Hong ${ }^{1,2}$, Cheol Kwak ${ }^{2,3}$ and Seok-Soo Byun ${ }^{1,2^{*}}$

\begin{abstract}
Background: There are limited data concerning patients treated with sequential bilateral kidney surgery. Current guidelines still lack an optimal surgical sequencing approach. We evaluated renal functional outcomes after sequential partial nephrectomy (PN) and radical nephrectomy (RN) in patients with bilateral renal cell carcinoma (RCC).

Methods: A propensity score matched cohort of 267 patients (synchronous bilateral RCCs, N=44 [88 lesions]; metachronous bilateral, $N=45$ [90 lesions]; unilateral, $N=178$ ) from two tertiary institutions were retrospectively analyzed. Synchronous bilateral RCCs were defined as diagnosis concomitantly or within 3 months of former tumor. Renal functional outcomes were defined as estimated glomerular filtration rate (eGFR) changes and de novo chronic kidney disease $(C K D$, stage $\geq 3)$ after surgery. Renal functional outcomes and clinical factors predicting de novo CKD were assessed using descriptive statistics and Cox regression analysis.

Results: In subgroup of bilateral RCCs, patients underwent sequential PN $(N=48)$, PN followed by RN $(N=8)$, or RN followed by PN ( $N=25)$. Final postoperative estimated glomerular filtration rates (eGFRs) were 79.4, 41.4, and 61.2 $\mathrm{ml} /$ minute/1.73 $\mathrm{m} 2$, respectively $(p=0.003)$. There were significant differences in eGFR decline from baseline and de novo chronic kidney disease (CKD stage $\geq \mathrm{III})$ among groups, with PN followed by RN group showing the worst functional outcomes (all $p<0.05$ ). Moreover, sequential PN subgroup in bilateral RCC showed significantly higher rate of de novo CKD than unilateral RCC group (13.8\% vs. 6.9\%, $p=0.016)$. On multivariate analysis, hypertension $(p=0.010)$ and surgery sequence (PN followed by RN, $p<0.001$ ) were significant predictors of de novo CKD.

Conclusions: The surgery sequence should be prudently determined in bilateral renal tumors. PN followed by RN showed a negative impact on renal functional preservation. Nephron-sparing surgery should be considered for all amenable bilateral RCCs.
\end{abstract}

Keywords: Bilaterality, Functional outcomes, Renal cell carcinoma, Partial nephrectomy, Sequence of surgery

\footnotetext{
* Correspondence: ssbyun@snubh.org

${ }^{\dagger} J u n g$ Kwon Kim and Hwanik Kim contributed equally to this work.

'Department of Urology, Seoul National University Bundang Hospital

(SNUBH), 166 Gumi-Ro, Bundang-gu, Seongnam-si, Gyeonggi-do 463-707,

Korea

2Department of Urology, Seoul National University College of Medicine, 103

Daehak-ro, Jongno-gu, Seoul 03080, Korea

Full list of author information is available at the end of the article
}

(C) The Author(s). 2021 Open Access This article is licensed under a Creative Commons Attribution 4.0 International License, which permits use, sharing, adaptation, distribution and reproduction in any medium or format, as long as you give appropriate credit to the original author(s) and the source, provide a link to the Creative Commons licence, and indicate if changes were made. The images or other third party material in this article are included in the article's Creative Commons. licence, unless indicated otherwise in a credit line to the material. If material is not included in the article's Creative Commons licence and your intended use is not permitted by statutory regulation or exceeds the permitted use, you will need to obtain permission directly from the copyright holder. To view a copy of this licence, visit http://creativecommons.org/licenses/by/4.0/ The Creative Commons Public Domain Dedication waiver (http://creativecommons.org/publicdomain/zero/1.0/) applies to the data made available in this article, unless otherwise stated in a credit line to the data. 


\section{Introduction}

It has been estimated that 73,800 new cases of cancers of the kidney and renal pelvis will be diagnosed in the United States in 2020 and that 14,800 people will die of them [1]. Approximately $85 \%$ of all kidney tumors are renal cell carcinoma (RCC). RCC occurs in bilateral kidneys, including synchronous RCC (diagnosed concomitantly or within 3 months of the former tumor) and metachronous RCC (tumor diagnosed 3 months after former tumor detection) in approximately $5 \%$ of all RCC patients [2-5]. Due to the relative rarity of bilateral presentation, even until now, there are limited data in the literature concerning patients treated with sequential bilateral kidney surgery. Few literatures have evaluated the functional impact of bilateral kidney surgery and how functional outcomes are influenced by tumor characteristics, modality selection, and patient-related risk factors [6-9].

Nevertheless, several studies have elucidated renal functional outcomes in patients with bilateral synchronous tumors who have undergone sequential bilateral kidney surgery. Simmons et al. have demonstrated that bilateral partial nephrectomy (PN) is associated with significantly improved estimated glomerular filtration rate (eGFR) compared to radical nephrectomy (RN) [6]. Packiam et al. have reported that patients for nonmetastatic bilateral synchronous tumors who have received simultaneous PN show lower mean postoperative 3 months ( $-6 \%$ vs. $-24 \%$, $p=0.015$ ) and median postoperative 12 months ( $-4 \%$ vs. $-22 \%, p<0.001)$ reduction in eGFR compared to staged (within 6 months) PN, respectively [7]. Singer et al. have observed that nephron-sparing surgery (NSS) enables dialysis to be avoided in more than $95 \%$ of patients [8]. Another study reported average $28.9 \%$ eGFR decline after treatment of both kidneys with 608 -day follow-up $(59 \rightarrow$ $41.9 \mathrm{ml} / \mathrm{min} / 1.73 \mathrm{~m}^{2}$ ) [9].

However, few studies have compared long term functional outcomes according to procedure sequence. Current guidelines still lack an optimal surgical sequencing approach [10-12]. Therefore, the objective of this study was to evaluate renal functional outcomes after sequential PN and RN in patients with bilateral RCC.

\section{Materials and methods}

\section{Ethics statement}

The Institutional Review Board of the Seoul National University Bundang Hospital approved this study (Approval number: B-2007-625-102). As the present study was carried out retrospectively, written informed consent from patients was waived. Personal identifiers were completely removed and data were analyzed anonymously. Our study was conducted according to ethical standards recommended by the 1964 Declaration of Helsinki and its later amendments.

\section{Study cohort}

From June 2003 to March 2018, a total of 267 patients (synchronous bilateral RCCs, $N=44$ [88 tumor lesions]; metachronous bilateral RCCs, $N=45$ [90 lesions]; unilateral RCCs, $N=178$ ) from two tertiary institutions (SNUH and SNUBH) were included in this study. Synchronous bilateral RCCs were defined as diagnoses concomitantly or within 3 months of the former tumor. Metachronous bilateral RCCs were defined as tumor diagnoses at intervals of at least 3 months. All included patients underwent surgery by open, laparoscopic, or robotic approach which was left to surgeon discretion, with curative intent in two centers [4-7]. Main operating surgeons determine the sequence of surgery under consideration of patients' clinical setting and multidisciplinary consultation. In general, treatment for the larger tumor was implemented first to allow better tumor control and the contralateral kidney to assist in recovery of renal function (Fig. 1). Variation in time between surgeries can be attributed to differences in recovery speed from initial surgery, patients' willingness to receive subsequent surgery, and preoperative general medical conditions. Patients were excluded if they had non-RCC histology or hereditary kidney diseases (e.g., the von Hippel-Lindau [VHL] disease, autosomal dominant polycystic kidney disease [ADPKD], Birt-Hogg-Dubé [BHD] syndrome, and so on). Clinical data in medical records were retrospectively reviewed [13].

\section{Acquisition and definition of data}

Clinical perioperative variables included age, gender, body mass index (BMI), past medical history (including diabetes mellitus [DM], hypertension [HTN], and chronic kidney disease [CKD]), symptoms at presentation, Eastern Cooperative Oncology Group (ECOG) performance status, and types of surgery (PN vs. RN). Pathological parameters including histological type according to the World Health Organization (WHO) classification system, pathologic stage according to the 8th edition of American Joint Committee guidelines, Fuhrman nuclear grade, and positive surgical margin were also evaluated [14, 15]. Tumor sizes are defined as the longest length of axes measured from pathologic specimen after surgery. Ischemic time is defined as warm ischemia time from first arterial clamping to first arterial unclamping. These are all monitored at the time of operation by main operating surgeon. Renal functional outcomes were defined as estimated glomerular filtration rate (eGFR) changes and incidence of de novo CKD (stage $\geq 3$ ) after surgery. eGFR changes at specific postoperative period was evaluated as follows: differences between postoperative 1 week eGFR and preoperative baseline eGFR, between postoperative 1 month eGFR and baseline eGFR, between postoperative 1 year eGFR 


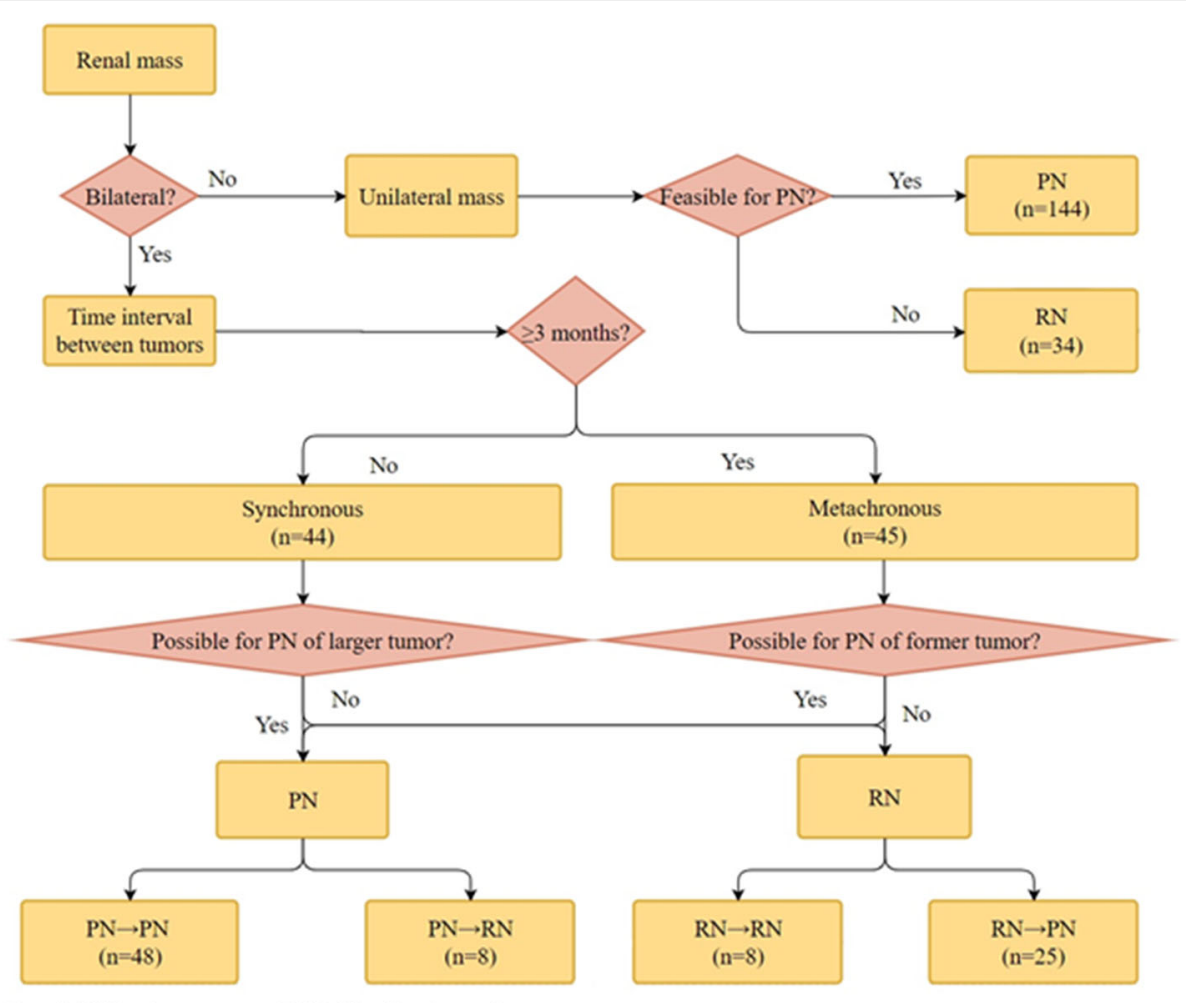

Partial Nephrectomy, RN: Radical nephrectomy

Fig. 1 Treatment algorithm

and baseline eGFR, and between latest eGFR and baseline eGFR. Renal functional evaluation was conducted using serum creatinine values obtained immediately before initial surgery, immediately before second surgery, and 3 to 6 months after the second surgery. Modification of Diet in Renal Disease equation was used to calculate eGFR [16]. CKD staging was conducted according to the National Kidney Foundation Disease Outcomes Quality Initiative Clinical Practice Guidelines [17].

\section{Follow-up protocol}

According to institutional standardized postoperative protocol, patients were generally followed-up after surgery at least every 6 months in the first year, annually during the next 4 years, and every 2 years thereafter. Follow-up protocols consisted of blood test including serum creatinine, computed tomography or magnetic resonance imaging studies, and chest radiography.

\section{Statistical analyses}

Clinicopathological characteristics were compared between unilateral and bilateral RCC groups using Chisquared test or Kruskal-Wallis test for categorical variables and one-way analysis of variance, independent $\mathrm{t}$ test or Mann-Whitney $U$ test for continuous variables as appropriate. Univariate and multivariate Cox- proportional hazard regression analyses were performed to evaluate significant variables associated with de novo CKD within postoperative 1 year and along entire follow-up period. Proportional hazard assumptions were evaluated using Schoenfeld test and log-log plot. The univariate results were used to determine the candidate variables for the final multivariate model in a backward model selection process. In all variables remaining in the final multivariate analysis, the $p$ value was set to 0.05 . Age, hypertension, pathologic tumor size, surgery sequence, and tumor chronology (only for Table 6) are variables included in the multivariate analysis at both Tables 5 and 6 . In addition, eGFR changes and clinical factors predicting de novo CKD in the propensity score matched cohort were assessed using descriptive statistics and Cox regression analysis [18]. The propensity score matching (PSM) was used to control other confounding factors when analyzing renal functional outcomes of unilateral and bilateral RCCs. The propensity scores matched using the nearest neighbor, 1:1 pair matching within 0.2 standard deviations (SDs) of the logit of the propensity score. By calculating the standardized differences of the means or proportions of each covariate after matching, baseline covariates with standardized differences $<0.1$ indicated a good balance between groups. Age, sex, DM, HTN, CKD, BMI, ECOG performance 
status, baseline eGFR, surgical type, expected blood loss, ischemic time, and pathologic tumor size was used as covariates in the PSM process. Subgroup analysis of bilateral RCC among synchronous and metachronous RCCs was also performed. All statistical analyses were performed using commercially available software IBM SPSS Statistics ver. 21.0 (Armonk, NY, USA and the statistical package for $\mathrm{R}$, ver. 2.13.2 (R Foundation for Statistical Computing [http://www.r-project.org/]). Twosided $p$ values $<0.05$ were considered statistically significant.

\section{Results}

Baseline characteristics between bilateral RCC group and unilateral RCC group are detailed in Table 1. Prepropensity table for the cohort before matching are detailed in supplementary Table 1 . There were no significant differences in baseline characteristics between groups after propensity score matching. Median time between surgeries in bilateral RCC group was 146.0 days (37.5 days for synchronous and 1899.0 days for metachronous subgroup). In the bilateral RCC cohort, subgroup comparative analysis for renal functional outcome (PN followed by $\mathrm{PN}$ vs. PN followed by $\mathrm{RN}$ vs. RN followed by PN group) was conducted (Table 2). Patients underwent sequential PN $(n=48)$, PN followed by RN $(n=8)$, or RN followed by PN $(n=25)$. Patients with sequential RN were excluded in the subgroup analysis due to intuitively prominent renal function deterioration compared to other groups. The mean eGFR follow-up duration was $65.6 \pm 47.6$ months (range, $0-166$ months). Final eGFR after bilateral surgery was $79.4 \pm 33.9,41.4 \pm$ 28.3 , and $61.2 \pm 29.8 \mathrm{ml} /$ minute $/ 1.73 \mathrm{~m}^{2}$ in these three groups, respectively $(p=0.003)$. There were significant differences in eGFR decline from baseline and de novo CKD among groups, with PN followed by RN group showing the worst functional outcomes (all $p<0.05$ ). PN followed by PN group had significantly higher latest eGFR $(p=0.003)$, less eGFR decline from baseline during the entire postoperative follow-up period (all $p<0.05$ ). They had also significantly less de novo CKD stage $\geq 3$ occurred within a year after 2nd operation $(p=0.036)$, less de novo CKD stage $\geq 3$ occurred after the one-year minimal follow-up period after 2nd operation (median/ mean follow-up period: 54.0/65.6 months, $p<0.001$ ), and less total number of patients with CKD at the last follow-up than other groups. RN followed by PN group had significantly less eGFR decline than PN followed by $\mathrm{RN}$ group at postoperative 1 week $(p=0.031)$ and the latest follow-up period ( $p=0.001)$ from baseline.

We also performed another subgroup analysis between bilateral synchronous RCC (PN followed by PN) subgroup and unilateral RCC (single PN) subgroup (Table 3). The bilateral synchronous RCC (PN followed by PN) subgroup

Table 1 Baseline characteristics after propensity score matching

\begin{tabular}{|c|c|c|c|c|}
\hline \multirow[t]{2}{*}{ Variables } & \multicolumn{2}{|c|}{$\begin{array}{l}\text { Bilateral RCC } \\
\text { ( } N=178 \text { lesions of } 89 \text { patients) }\end{array}$} & \multirow{2}{*}{$\begin{array}{l}\text { Unilateral } \\
\text { RCC } \\
\text { ( } N=178 \\
\text { lesions of } \\
178 \\
\text { patients) }\end{array}$} & \multirow[t]{2}{*}{$\begin{array}{l}P \\
\text { value }^{\S}\end{array}$} \\
\hline & $\begin{array}{l}\text { Synchronous lesion } \\
(N=88)\end{array}$ & $\begin{array}{l}\text { Metachronous lesion } \\
(N=90)\end{array}$ & & \\
\hline Age, mean (SD) & $54.0 \pm 12.7$ & $55.9 \pm 12.9$ & $54.2 \pm 13.4$ & 0.549 \\
\hline Sex, male, N (\%) & $74(84.1 \%)$ & $74(82.2 \%)$ & $142(79.8 \%)$ & 0.856 \\
\hline DM, yes, N (\%) & $17(19.3 \%)$ & $18(20.0 \%)$ & $22(16.7 \%)$ & 0.792 \\
\hline HTN, yes, N (\%) & $47(53.4 \%)$ & $42(46.7 \%)$ & 89 (50.0\%) & 0.564 \\
\hline BMI, mean (SD) & $25.0 \pm 3.7$ & $24.2 \pm 3.1$ & $24.7 \pm 3.0$ & 0.367 \\
\hline ECOG performance status, N (\%) & & & & 0.415 \\
\hline$\leq 1$ & $86(97.7 \%)$ & $86(95.6 \%)$ & $167(93.8 \%)$ & \\
\hline$\geq 2$ & $2(2.3 \%)$ & $4(4.4 \%)$ & $11(6.2)$ & \\
\hline $\begin{array}{l}\text { Surgical type, N (\%) } \\
\text { (Open / Laparoscopic / Robotic) }\end{array}$ & $\begin{array}{l}37(42.0 \%) / \\
13(14.8 \%) / \\
38(43.2 \%)\end{array}$ & $\begin{array}{l}44(48.9 \%) / \\
9(10.0 \%) / \\
37(41.1 \%)\end{array}$ & $\begin{array}{l}94(52.8 \%) / \\
11(6.2 \%) / \\
73(41.0 \%)\end{array}$ & 0.183 \\
\hline EBL, ml, mean (SD) & $249.1 \pm 107.5$ & $259.0 \pm 217.9$ & $226.1 \pm 261.5$ & 0.878 \\
\hline Ischemic time, min, mean (SD) ${ }^{a}$ & $21.5 \pm 9.8$ & $22.2 \pm 10.7$ & $21.4 \pm 16.3$ & 0.904 \\
\hline Pathologic tumor size, mm, mean (SD) & $33.5 \pm 34.8$ & $32.1 \pm 27.6$ & $32.8 \pm 23.3$ & 0.949 \\
\hline Baseline eGFR, ml/min/1.73 m², mean (SD) & $71.6 \pm 32.5$ & $76.0 \pm 25.4$ & $78.6 \pm 22.3$ & 0.224 \\
\hline Baseline $C K D$, stage $\geq 3$ & $28(31.8 \%)$ & $16(17.8 \%)$ & 39 (21.9\%) & 0.068 \\
\hline
\end{tabular}

$\S$ Evaluates differences among all 3 groups

a Partial nephrectomy only 
Table 2 Functional outcome of bilateral RCC cohort regardless of time interval between tumors

\begin{tabular}{|c|c|c|c|c|}
\hline Variables & $\mathrm{PN} \rightarrow \mathrm{PN}(N=48)$ & $\begin{array}{l}\mathrm{PN} \rightarrow \mathrm{RN} \\
(N=8)\end{array}$ & $\begin{array}{l}\mathrm{RN} \rightarrow \mathrm{PN} \\
(N=25)\end{array}$ & $P$ value ${ }^{\S}$ \\
\hline Preoperative eGFR, ml/min/1.73 $\mathrm{m}^{2}$, mean (SD) (before 1st surgery) & $84.7 \pm 25.4$ & $83.1 \pm 19.6$ & $79.6 \pm 33.4$ & 0.759 \\
\hline \multirow[t]{2}{*}{ Latest eGFR, $\mathrm{ml} / \mathrm{min} / 1.73 \mathrm{~m}^{2}$, mean (SD) } & $79.4 \pm 33.9$ & $41.4 \pm 28.3$ & $61.2 \pm 29.8$ & 0.003 \\
\hline & & $p=0.108^{a}$ & & \\
\hline \multicolumn{5}{|l|}{ eGFR decline from baseline, mean (SD) } \\
\hline \multirow[t]{2}{*}{ Postoperative 1 week (after 2nd surgery) } & $6.9 \pm 18.5$ & $28.5 \pm 18.2$ & $10.6 \pm 16.5$ & 0.009 \\
\hline & & $p=0.031^{a}$ & & \\
\hline \multirow[t]{2}{*}{ Postoperative 1 month (after 2nd surgery) } & $5.1 \pm 15.0$ & $28.7 \pm 23.2$ & $9.9 \pm 13.2$ & 0.001 \\
\hline & & $p=0.058^{a}$ & & \\
\hline \multirow[t]{2}{*}{ Postoperative 1 year (after 2nd surgery) } & $5.8 \pm 17.5$ & $29.4 \pm 24.2$ & $31.3 \pm 6.3$ & 0.018 \\
\hline & & $p=0.035^{a}$ & & \\
\hline \multirow[t]{2}{*}{ Latest } & $-0.64 \pm 22.5$ & $30.5 \pm 26.6$ & $-2.7 \pm 21.3$ & 0.001 \\
\hline & & $p=0.001^{a}$ & & \\
\hline \multicolumn{5}{|l|}{ De novo $C K D$, stage $\geq 3$} \\
\hline Baseline CKD, before 1st surgery & $8(16.7 \%)$ & $1(12.5 \%)$ & $5(20.0 \%)$ & 0.874 \\
\hline Baseline CKD, before 2nd surgery & $6(12.5 \%)$ & $1(12.5 \%)$ & $9(36.0 \%)$ & 0.049 \\
\hline De novo, $\leq 1$ year after 2 nd surgery & $9(18.8 \%)$ & $4(50 \%)$ & $5(20 \%)$ & 0.036 \\
\hline De novo, $>1$ year after 2 nd surgery & $0(0 \%)$ & $2(25.0 \%)$ & $1(4.0 \%)$ & $<0.001$ \\
\hline Total number of CKD patients last follow-up & $15(31.3 \%)$ & $7(87.5 \%)$ & $15(60 \%)$ & $<0.001$ \\
\hline
\end{tabular}

SD Standard Deviation

eGFR follow-up duration (mean \pm SD): $65.6 \pm 47.6$ months (range, 0-166 months)

8 patients with RN followed by RN were excluded from the analysis

$\S$ Evaluates differences among all surgery groups at specific points

a Evaluates differences between 'PN $\rightarrow \mathrm{RN}^{\prime}$ group and 'RN $\rightarrow \mathrm{PN}^{\prime}$ group

had no significant difference in eGFR decline at the latest follow-up period from baseline compared to the unilateral RCC (single PN) subgroup $(p=0.770)$, although it had higher de novo CKD rate until postoperative 1 year and during the entire follow-up period $(13.8 \%$ vs. $6.9 \%, p=$ 0.016). Additional subgroup analysis between bilateral synchronous and metachronous RCC (PN followed by
PN) subgroups revealed no significant differences in variables among groups (Table 4).

Multivariate analysis for the prediction of de novo CKD until postoperative 1 year revealed that hypertension (Hazard ratio [HR]: 2.159, 95\% Confidence Interval [CI]: $1.233-3.783, p=0.007)$, pathologic tumor size at 1st surgery (HR: 1.012, 95\% CI: 1.006-1.024, $p=0.010$ )

Table 3 Comparative analysis between bilateral synchronous tumor subgroup (PN followed by PN) and unilateral RCC (single PN)

\begin{tabular}{|c|c|c|c|}
\hline Variables & $\begin{array}{l}\text { Bilateral } \\
\text { ( } N=58 \text { lesions of } \\
29 \text { patients) }\end{array}$ & $\begin{array}{l}\text { Unilateral } \\
\text { ( } N=144 \text { lesions of } 144 \text { patients) }\end{array}$ & $P$ value \\
\hline Preoperative eGFR, ml/min $/ 1.73 \mathrm{~m}^{2}$, mean (SD) & $78.2 \pm 24.3$ & $82.2 \pm 22.9$ & 0.106 \\
\hline aLatest eGFR, ml/min/1.73 m², mean (SD) & $80.2 \pm 22.7$ & $83.1 \pm 20.4$ & 0.182 \\
\hline \multicolumn{4}{|l|}{ eGFR decline from baseline, mean (SD) } \\
\hline Postoperative 1 week & $7.2 \pm 16.1$ & $-3.5 \pm 15.4$ & $<0.001$ \\
\hline Postoperative 1 month & $3.5 \pm 14.6$ & $-1.7 \pm 15.5$ & 0.037 \\
\hline Postoperative 1 year & $5.3 \pm 17.3$ & $0.9 \pm 16.3$ & 0.099 \\
\hline bLatest & $-2.0 \pm 23.8$ & $-1.0 \pm 18.5$ & 0.770 \\
\hline De novo $C K D, \leq 1$ year & $8(13.8 \%)$ & $8(7.5 \%)$ & 0.022 \\
\hline De novo CKD, total & $8(13.8 \%)$ & $10(6.9 \%)$ & 0.016 \\
\hline
\end{tabular}

aLatest eGFR: The very last estimated Glomerular Filtration Rate measured during the follow-up period

batest: The last time a patient was followed-up and evaluated eGFR 
Table 4 Comparative analysis between PN followed by PN subgroups (synchronous vs. metachronous) [latter surgery]

\begin{tabular}{|c|c|c|c|}
\hline Variables & $\begin{array}{l}\text { Synchronous } \\
\text { (N=29 patients) }\end{array}$ & $\begin{array}{l}\text { Metachronous } \\
(N=19 \text { patients })\end{array}$ & $P$ value \\
\hline Preoperative eGFR, ml/min/1.73 m², mean (SD) & $76.5 \pm 22.4$ & $82.1 \pm 25.1$ & 0.434 \\
\hline${ }^{a}$ Latest eGFR, $\mathrm{ml} / \mathrm{min} / 1.73 \mathrm{~m}^{2}$, mean (SD) & $80.2 \pm 24.0$ & $78.1 \pm 31.2$ & 0.247 \\
\hline \multicolumn{4}{|l|}{ eGFR decline from baseline, mean (SD) } \\
\hline Postoperative 1 week & $6.5 \pm 18.0$ & $7.4 \pm 19.7$ & 0.871 \\
\hline Postoperative 1 month & $3.0 \pm 14.9$ & $8.4 \pm 15.1$ & 0.230 \\
\hline Postoperative 1 year & $3.5 \pm 17.5$ & $9.3 \pm 17.5$ & 0.266 \\
\hline b Latest & $-3.7 \pm 24.1$ & $4.0 \pm 19.5$ & 0.250 \\
\hline \multicolumn{4}{|l|}{ De novo $C K D$, stage $\geq 3$} \\
\hline Baseline CKD, Before 1st surgery & $7(24.1 \%)$ & $1(5.3 \%)$ & 0.123 \\
\hline Baseline CKD, Before 2nd surgery & $5(17.2 \%)$ & $1(5.3 \%)$ & 0.381 \\
\hline De novo, $\leq 1$ year after 2 nd surgery & $5(17.2 \%)$ & $4(21.1 \%)$ & 1.000 \\
\hline De novo, $>1$ year after 2 nd surgery & $0(0 \%)$ & $0(0 \%)$ & 1.000 \\
\hline Total number of pts. with CKD at last follow-up & $10(34.5 \%)$ & $5(26.3 \%)$ & 0.751 \\
\hline
\end{tabular}

aLatest eGFR: The very last estimated Glomerular Filtration Rate measured during the follow-up period

batest: The last time a patient was followed-up and evaluated eGFR

Table 5 Multivariate Cox regression analyses of variables associated with de novo CKD within postoperative one year

\begin{tabular}{|c|c|c|c|c|c|c|}
\hline & \multicolumn{3}{|c|}{ Univariate analysis } & \multicolumn{3}{|c|}{ Multivariate analysis } \\
\hline & HR & $95 \% \mathrm{Cl}$ & $P$ value & HR & $95 \% \mathrm{Cl}$ & $P$ value \\
\hline Age (years) & 1.032 & $1.009-1.056$ & 0.001 & 1.036 & $1.011-1.062$ & 0.005 \\
\hline BMl & 1.011 & $0.924-1.106$ & 0.810 & & & \\
\hline Sex, male & 0.711 & $0.348-1.454$ & 0.350 & & & \\
\hline DM & 1.295 & $0.646-2.595$ & 0.466 & & & \\
\hline HTN & 2.413 & $1.353-4.304$ & 0.003 & 2.159 & $1.233-3.783$ & 0.007 \\
\hline Baseline eGFR & 0.992 & $0.982-1.003$ & 0.150 & & & \\
\hline Baseline $\mathrm{Hb}$ & 0.986 & $0.875-1.112$ & 0.822 & & & \\
\hline EBL (1st surgery) & 1.000 & $0.999-1.001$ & 0.799 & & & \\
\hline EBL (2nd surgery) & 1.000 & $0.999-1.001$ & 0.663 & & & \\
\hline Ischemic time (1st surgery) & 1.005 & $0.974-1.037$ & 0.763 & & & \\
\hline Ischemic time (2nd surgery) & 0.987 & $0.957-1.019$ & 0.431 & & & \\
\hline Pathologic tumor size (1st surgery) & 1.015 & $1.006-1.024$ & 0.001 & 1.012 & $1.003-1.021$ & 0.010 \\
\hline Pathologic tumor size (2nd surgery) & 1.004 & $0.996-1.011$ & 0.326 & & & \\
\hline Time between operations & 1.000 & $1.000-1.001$ & 0.083 & & & \\
\hline \multicolumn{7}{|l|}{ Surgery sequence } \\
\hline $\mathrm{PN} \rightarrow \mathrm{PN}$ & Reference & & & Reference & & \\
\hline $\mathrm{PN} \rightarrow \mathrm{RN}$ & 2.249 & $1.088-4.786$ & 0.006 & 1.837 & $1.028-3.635$ & 0.007 \\
\hline $\mathrm{RN} \rightarrow \mathrm{PN}$ & 1.510 & $1.096-5.750$ & 0.030 & 1.235 & $0.947-5.276$ & 0.066 \\
\hline PN (unilateral case) & 0.663 & $0.285-1.543$ & 0.341 & 0.315 & $0.119-0.832$ & 0.261 \\
\hline \multicolumn{7}{|l|}{ Tumor chronology } \\
\hline Synchronous & Reference & & & & & \\
\hline Metachronous & 1.370 & $0.660-2.840$ & 0.398 & & & \\
\hline \multicolumn{7}{|l|}{ Tumor multiplicity } \\
\hline Unilateral & Reference & & & & & \\
\hline Bilateral & 1.244 & $0.698-2.215$ & 0.459 & & & \\
\hline
\end{tabular}


and PN followed by RN sequence (HR: 1.837, 95\% CI: $1.028-3.635, p=0.007$ ) were significant factors (Table 5). A multivariate analysis for the prediction of de novo CKD during the entire period revealed that hypertension (HR: 1.905, 95\% CI: 1.172-3.265, $p=0.010$ ), PN followed by RN sequence (HR: 1.888, 95\% CI: 1.088-4.055, $p<0.001$ ), and metachronous RCC (HR: 2.682, 95\% CI: 1.032-6.973, $p=0.043)$ were significant predictive factors (Table 6). The difference in time interval between tumor occurrences in metachronous RCC was not significantly related to de novo CKD incidence $(p=0.083$ for de novo CKD within postoperative 1 year and $p=$ 0.056 for de novo CKD during the entire follow-up period).

\section{Discussion}

Surgical management of RCC ultimately aims to balance numerous considerations with attempts to minimize operative morbidity and decline of renal function. To this end, when technically feasible, NSS has been advocated to maximize preservation of renal parenchyma and avoid the incidence and sequelae of renal function deterioration [19-22]. In the setting of bilateral renal masses, guidelines favor the performance of bilateral $\mathrm{PN}$ when it is technically feasible [10-12]. However, there is only a short reference to this simple guideline statement without specific details on how to do it in real clinical practice. For example, in case of the situation that both RN and PN are inevitably needed, it is a dilemma to decide which procedure has to be done first to achieve satisfactory renal function preservation. Nevertheless, using our prospectively collected database, we proved that doing RN first followed by PN sequence would be better in terms of preserving renal function than proceeding in reverse order. According to our multivariate analysis, hypertension and PN followed by RN sequence are commonly independent risk factors for de novo CKD within postoperative 1 year and the entire follow-up period. We also identified evidence in other literature supporting our findings. Krohn et al. have reported that 1 year after

Table 6 Multivariate Cox regression analyses of variables associated with de novo CKD during the entire follow-up period

\begin{tabular}{|c|c|c|c|c|c|c|}
\hline & \multicolumn{3}{|c|}{ Univariate analysis } & \multicolumn{3}{|c|}{ Multivariate analysis } \\
\hline & HR & $95 \% \mathrm{Cl}$ & $P$ value & HR & $95 \% \mathrm{Cl}$ & $P$ value \\
\hline Age (years) & 1.031 & $1.009-1.053$ & 0.005 & 1.005 & $0.971-1.040$ & 0.780 \\
\hline BMl & 1.029 & $0.946-1.120$ & 0.500 & & & \\
\hline Sex, male & 0.607 & $0.306-1.205$ & 0.154 & & & \\
\hline DM & 1.530 & $0.806-2.905$ & 0.194 & & & \\
\hline HTN & 1.956 & $1.147-3.335$ & 0.014 & 1.905 & $1.172-3.265$ & 0.010 \\
\hline Baseline eGFR & 0.992 & $0.982-1.002$ & 0.106 & & & \\
\hline Baseline $\mathrm{Hb}$ & 1.002 & $0.902-1.114$ & 0.969 & & & \\
\hline EBL (1st surgery) & 1.000 & $0.999-1.000$ & 0.784 & & & \\
\hline EBL (2nd surgery) & 1.000 & $0.999-1.001$ & 0.680 & & & \\
\hline Ischemic time (1st surgery) & 1.001 & $0.972-1.032$ & 0.921 & & & \\
\hline Ischemic time (2nd surgery) & 0.985 & $0.956-1.014$ & 0.301 & & & \\
\hline Pathologic tumor size (1st surgery) & 1.016 & $1.007-1.025$ & 0.001 & 1.013 & $1.000-1.027$ & 0.051 \\
\hline Pathologic tumor size (2nd surgery) & 1.004 & $0.997-1.011$ & 0.278 & & & \\
\hline Time between operations & 1.000 & $1.000-1.001$ & 0.056 & & & \\
\hline \multicolumn{7}{|l|}{ Surgery sequence } \\
\hline $\mathrm{PN} \rightarrow \mathrm{PN}$ & Reference & & & Reference & & \\
\hline $\mathrm{PN} \rightarrow \mathrm{RN}$ & 2.919 & $1.386-6.146$ & 0.001 & 1.888 & $1.088-4.055$ & $<0.001$ \\
\hline $\mathrm{RN} \rightarrow \mathrm{PN}$ & 2.190 & $1.165-4.114$ & 0.015 & 1.041 & $1.003-3.258$ & 0.103 \\
\hline PN (unilateral case) & 0.782 & $0.366-1.669$ & 0.525 & 0.233 & $0.088-0.614$ & 0.718 \\
\hline \multicolumn{7}{|l|}{ Tumor chronology } \\
\hline Synchronous & Reference & & & Reference & & \\
\hline Metachronous & 2.250 & $1.121-4.516$ & 0.023 & 2.682 & $1.032-6.973$ & 0.043 \\
\hline \multicolumn{7}{|l|}{ Tumor multiplicity } \\
\hline Unilateral & Reference & & & & & \\
\hline Bilateral & 1.421 & $0.824-2.449$ & 0.206 & & & \\
\hline
\end{tabular}


donation, the remained kidney manages to compensate up to $70 \%$ of renal function before surgery [23]. A plausible explanation is that in the remained kidney, vasodilation and increased renal plasma flow can occur immediately after surgery. These changes, combined with the process of glomerular hypertrophy, can increase glomerular filtration of the remnant kidney by approximately $40 \%$ without occurring a concomitant increase in glomerular capillary pressure [24-26]. This adaptive hyperfiltration also occurs in older kidney donors, although more modestly than in younger donors [27]. Taner et al. have reported that compensatory hypertrophy and GFR increase can occur in the remaining kidney of medically complex living donors at a comparable rate to those of standard donors [28]. These findings confirmed reassurance for delicately selected medically complex living donors. One study has concluded that RCC is not an independent risk factor for renal function decrease after nephrectomy. RCC patients with few morbidities could have the same deterioration of meanly $30 \%$ of kidney function compared with living donors. However, their lower baseline function can result in an increased risk for CKD [29]. These findings imply that patients with $\mathrm{RN}$ who have sufficient period to compensate for their renal function until $\mathrm{PN}$ can preserve favorable renal function without risk for CKD.

$\mathrm{PN}$ is generally related to a lower risk of developing clinically significant CKD than RN. Postoperative impairment in kidney function occurs most commonly in the first year after nephrectomy and appears stable over time. Age, Tumor stage, and preoperative kidney function are predictors of incident CKD after kidney cancer operation [30]. Bilateral PN needs more careful consideration compared to ipsilateral $\mathrm{PN}$ due to potential additional loss of renal function secondary to bilateral renal ischemia from hemorrhage, hypotension, and prolonged operative time [19]. These challenges have made some surgeons support staged bilateral PN as opposed to simultaneous bilateral $\mathrm{PN}$ in a single setting. PN, if performed well, is also a possible choice for larger renal tumors as it generates tolerable surgical morbidity, better renal function preservation, and equivalent cancer control with potential for better long-term survival than RN [31]. Bercz et al. showed acceptable oncological and functional outcomes from 65 patients. They reported significant postoperative renal function deterioration (44.8\% eGFR decrease for synchronous (mostly RN $\rightarrow$ $\mathrm{PN}$ ) and 30.4\% decrease for metachronous tumor (all $\mathrm{RN} \rightarrow \mathrm{PN}$ ) after the second operation, respectively), but hemodialysis was rarely required. Compared to their results, we showed more eGFR preservation if $\mathrm{RN} \rightarrow \mathrm{PN}$ (23.1\% decrease) is performed respectively even though their cohort characteristics are slightly different [32].
This study has some limitations. First, even with two large tertiary centers' cohort, the study population was still small due to the rarity of bilateral RCCs with its retrospective nature. In addition, there was no analysis of differences in renal functions that might occur due to the heterogeneity of surgical techniques and different time intervals of operation for bilateral metachronous RCC. Thirdly, our result from eight patients that PN followed by RN can negatively affect renal function preservation is necessary to be verified in further studies with larger numbers of patients. Finally, it was difficult to identify genetic differences between these patients. These issues can be elucidated if multicenter prospective randomized clinical trials are performed in the near future. Nevertheless, to the best of our knowledge, the current study is the first of its kind that assesses functional aspects of both kidney cancer surgery performed in large institutions over a relatively long period of time. This is also the first study to find that $\mathrm{RN}$ followed by $\mathrm{PN}$ is superior in terms of preserving renal functions in the opposite order by evaluating cohorts through propensity score matching analysis.

\section{Conclusion}

This study evaluated renal functional outcomes of bilateral RCC patients after sequential kidney cancer surgery in a large contemporary cohort. The sequence of surgery should be prudently determined in bilateral renal tumors. The best ideal scenario is PN for both tumors. However, it is essential to make decisions about surgery sequence in the inevitable situation of performing a combination of radical and partial nephrectomy. PN followed by RN showed a negative impact on renal functional preservation. Thus, NSS should be considered for all amenable bilateral RCCs.

\section{Abbreviations \\ ADPKD: Autosomal dominant polycystic kidney disease; BHD: Birt-Hogg- Dubé syndrome; BMI: Body mass index; CKD: Chronic kidney disease; DM: Diabetes mellitus; EBL: Expected blood loss; eGFR: Estimated glomerular filtration rate; HTN: Hypertension; NSS: Nephron sparing surgery; PN: Partial nephrectomy; PSM: Propensity score matching; RCC: Renal cell carcinoma; RN: Radical nephrectomy; VHL: Von Hippel-Lindau disease}

\section{Supplementary Information}

The online version contains supplementary material available at https://doi. org/10.1186/s12885-021-08324-3.

Additional file 1: Supplementary Table 1. Baseline characteristics before propensity score matching.

\section{Acknowledgments}

None.

\section{Authors' contributions}

J.K.K. and H.K. contributed equally to the work and should be considered cofirst authors. Conceptualization, J.K.K., H.K. and S.S.B.; methodology, J.K.K. and S.S.B.; software, J.K.K.; validation, H.K., S.L., H.L., J.J.O., S.K.H., C.K. and S.S.B.; 
formal analysis, J.K.K., H.K., and S.S.B.; investigation, J.K.K., H.K. and S.K.H.; resources, J.K.K., S.L., H.L., S.K.H. C.K. and S.S.B.; data curation, J.K.K.; writing-original draft preparation, J.K.K., H.K. and S.S.B.; writing-review and editing, J.K.K., H.K., S.L., H.L., J.J.O., S.K.H., C.K. and S.S.B.; visualization, J.K.K. and H.K.; supervision S.S.B.; project administration, H.K., J.K.K., and S.S.B.; funding acquisition, none. All authors have read and agreed to the published version of the manuscript.

\section{Funding}

The authors received no specific funding for this work.

\section{Availability of data and materials}

The datasets used and /or analyzed during the current study are available from the corresponding on reasonable request.

\section{Declarations}

\section{Ethics approval and consent to participate}

This study was approved by the institutional review board of Seoul National University Bundang Hospital (Approval number: B-2007-625-102). The patients' consent was waived due to the retrospective nature and minimal risk to the subjects approved by the institutional review board of Seoul National University Bundang Hospital. Our study was conducted according to ethical standards recommended by the 1964 Declaration of Helsinki and its later amendments.

\section{Consent for publication}

Not applicable.

\section{Competing interests}

The authors declare that they have no competing interests.

\section{Author details}

'Department of Urology, Seoul National University Bundang Hospital (SNUBH), 166 Gumi-Ro, Bundang-gu, Seongnam-si, Gyeonggi-do 463-707, Korea. ${ }^{2}$ Department of Urology, Seoul National University College of Medicine, 103 Daehak-ro, Jongno-gu, Seoul 03080, Korea. ${ }^{3}$ Department of Urology, Seoul National University Hospital (SNUH), Seoul, Korea.

Received: 28 January 2021 Accepted: 6 May 2021

Published online: 24 May 2021

\section{References}

1. Siegel RL, Miller KD, Jemal A. Cancer statistics, 2020. CA Cancer J Clin. 2020; 70(1):7-30. https://doi.org/10.3322/caac.21590.

2. Wiklund F, Tretli S, Choueiri TK, Signoretti S, Fall K, Adami HO. Risk of bilateral renal cell cancer. J Clin Oncol. 2009;27(23):3737-41. https://doi. org/10.1200/JCO.2008.20.6524.

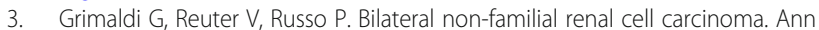
Surg Oncol. 1998;5(6):548-52. https://doi.org/10.1007/BF02303649.

4. Lowrance WT, Yee DS, Maschino AC, Cronin AM, Bernstein M, Thompson $\mathrm{RH}$, et al. Developments in the surgical management of sporadic synchronous bilateral renal tumours. BJU Int. 2010;105(8):1093-7. https://doi. org/10.1111/j.1464-410X.2009.08844.x.

5. Sheikh NA, Khan MH, Pillai S, Lang S, Nabi G. Outcomes of synchronous and metachronous bilateral small renal masses $(<4 \mathrm{~cm})$ : a population-based cohort study. Int Urol Nephrol. 2018;50(4):657-63. https://doi.org/10.1007/ s11255-018-1817-x.

6. Simmons MN, Brandina R, Hernandez AV, et al. Surgical management of bilateral synchronous kidney tumors: functional and oncological outcomes. J Urol. 2010;184(3):865-72. https://doi.org/10.1016/j.juro.2010.05.042.

7. Packiam VT, Tsivian M, Lohse CM, et al. Simultaneous versus staged partial nephrectomies for bilateral synchronous solid renal masses. Urol Oncol. 2020;38(7):640.e13-22.

8. Singer EA, Vourganti S, Lin KY, Gupta GN, Pinto PA, Rastinehad AR, et al. Outcomes of patients with surgically treated bilateral renal masses and a minimum of 10 years of follow up. J Urol. 2012;188(6):2084-8. https://doi. org/10.1016/j.juro.2012.08.038.

9. Woodson B, Fernandez R, Stewart C, Mandava S, Wang L, Lee BR. Bilateral synchronous sporadic renal masses: intermediate functional and oncological outcomes at a single institution. Int Urol Nephrol. 2013;45(3):619-25. https:// doi.org/10.1007/s11255-013-0414-2.

10. Campbell S, Uzzo RG, Allaf ME, Bass EB, Cadeddu JA, Chang A, et al. Renal mass and localized renal cancer: AUA guideline. J Urol. 2017;198(3):520-9. https://doi.org/10.1016/j.juro.2017.04.100.

11. Ljungberg B, Albiges $L$, Abu-Ghanem $Y$, Bensalah K, Dabestani S, FernándezPello $\mathrm{S}$, et al. European association of urology guidelines on renal cell carcinoma: the 2019 update. Eur Urol. 2019;75(5):799-810. https://doi.org/1 0.1016/j.eururo.2019.02.011.

12. Motzer RJ, Jonasch E, Boyle S, Carlo MI, Manley B, Agarwal N, et al. NCCN guidelines insights: kidney cancer, version 1.2021. J Natl Compr Cancer Netw. 2020;18(9):1160-70. https://doi.org/10.6004/jnccn.2020.0043.

13. Kim JK, Lee H, Oh JJ, et al. Synchronous bilateral RCC is associated with poor recurrence-free survival compared with unilateral RCC: a single-center study with propensity score matching analysis. Clin Genitourin Cancer. 2019; 17(3):e570-80

14. Lopez-Beltran A, Scarpelli M, Montironi R, Kirkali Z. 2004 WHO classification of the renal tumors of the adults. Eur Urol. 2006;49(5):798-805. https://doi. org/10.1016/j.eururo.2005.11.035.

15. Amin MB, Edge SB, Greene FL, et al. AJCC Cancer Staging Manual. 8th ed. New York: Springer; 2017. p. 12

16. Lin J, Knight EL, Hogan ML, Singh AK. A comparison of prediction equations for estimating glomerular filtration rate in adults without kidney disease. J Am Soc Nephrol. 2003;14(10):2573-80. https://doi.org/10.1097/01.ASN. 0000088721.98173.4B

17. Steinberg EP, Eknoyan G, Levin NW, Eschbach JW, Golper TA, Owen WF, et al. Methods used to evaluate the quality of evidence underlying the national kidney foundation-dialysis outcomes quality initiative clinical practice guidelines: description, findings, and implications. Am J Kidney Dis. 2000;36(1):1-11. https://doi.org/10.1053/ajkd.2000.8233.

18. D'Agostino RB Jr. Propensity score methods for bias reduction in the comparison of a treatment to a non-randomized control group. Stat Med. 1998;17(19):2265-81. https://doi.org/10.1002/(SICI)1097-0258(19981015)17:1 9<2265*:AID-SIM918>3.0.CO $2-$ B

19. Novick AC, Streem S, Montie JE, Pontes JE, Siegel S, Montague DK, et al. Conservative surgery for renal cell carcinoma: a single-center experience with 100 patients. J Urol. 1989;141(4):835-9. https://doi.org/10.1016/500225347(17)41026-3.

20. Go AS, Chertow GM, Fan D, McCulloch CE, Hsu C. Chronic kidney disease and the risks of death, cardiovascular events, and hospitalization. N Engl J Med. 2004;351(13):1296-305. https://doi.org/10.1 056/NEJMoa041031.

21. Tonelli M, Wiebe N, Culleton B, House A, Rabbat C, Fok M, et al. Chronic kidney disease and mortality risk: a systematic review. JAm Soc Nephrol JASN. 2006;17(7):2034-47. https://doi.org/10.1681/ASN.2005101085.

22. Mir MC, Ercole C, Takagi T, Zhang Z, Velet L, Remer EM, et al. Decline in renal function after partial nephrectomy: etiology and prevention. J Urol. 2015;193(6):1889-98. https://doi.org/10.1016/j.juro.2015.01.093.

23. Krohn AG, Ogden DA, Holmes JH. Renal function in 29 healthy adults before and after nephrectomy. JAMA. 1966;196(4):322-4. https://doi.org/10.1 001/jama.1966.03100170064019.

24. Lenihan CR, Busque S, Derby G, Blouch K, Myers BD, Tan JC. Longitudinal study of living kidney donor glomerular dynamics after nephrectomy. J Clin Invest. 2015;125(3):1311-8. https://doi.org/10.1172/JCl78885.

25. Rook M, Hofker HS, van Son WJ, van der Heide JJ H, Ploeg RJ, Navis GJ. Predictive capacity of pre-donation GFR and renal reserve capacity for donor renal function after living kidney donation. Am J Transplant. 2006; 6(7):1653-9. https://doi.org/10.1111/j.1600-6143.2006.01359.x.

26. Burballa C, Crespo M, Redondo-Pachón D, Pérez-Sáez MJ, Arias-Cabrales C Mir $\mathrm{M}$, et al. Factors associated with renal function compensation after donor nephrectomy. Nefrologia. 2018;38(5):528-34. https://doi.org/10.1016/j. nefro.2018.02.008

27. Velosa JA, Offord KP, Schroeder DR. Effect of age, sex, and glomerular filtration rate on renal function outcome of living kidney donors. Transplantation. 1995;60:1618.

28. Taner T, lqbal CW, Textor SC, Stegall MD, Ishitani MB. Compensatory hypertrophy of the remaining kidney in medically complex living kidney donors over the long term. Transplantation. 2015:99(3):555-9. https://doi. org/10.1097/TP.0000000000000356

29. Timsit MO, Nguyen KN, Rouach Y, Elie C, Loupy A, Fournier C, et al. Kidney function following nephrectomy: similitude and discrepancies between 
kidney cancer and living donation. Urol Oncol. 2012;30(4):482-6. https://doi. org/10.1016/j.urolonc.2010.04.005.

30. Leppert JT, Lamberts RW, Thomas IC, Chung BI, Sonn GA, Skinner EC, et al. Incident CKD after radical or partial nephrectomy. J Am Soc Nephrol. 2018; 29(1):207-16. https://doi.org/10.1681/ASN.2017020136.

31. Mir MC, Derweesh I, Porpiglia F, Zargar H, Mottrie A, Autorino R. Partial nephrectomy versus radical nephrectomy for clinical $\mathrm{T} 1 \mathrm{~b}$ and $\mathrm{T} 2$ renal tumors: a systematic review and meta-analysis of comparative studies. Eur Urol. 2017;71(4):606-17. https://doi.org/10.1016/j.eururo.2016.08.060

32. Bercz C, Thomas B, Basco Z, Berczi C, Thomas B, Bacso Z, et al. Bilateral renal cancers: oncological and functional outcomes. Int Urol Nephrol. 2016;48(10): 1617-22. https://doi.org/10.1007/s11255-016-1354-4.

\section{Publisher's Note}

Springer Nature remains neutral with regard to jurisdictional claims in published maps and institutional affiliations.

Ready to submit your research? Choose BMC and benefit from:

- fast, convenient online submission

- thorough peer review by experienced researchers in your field

- rapid publication on acceptance

- support for research data, including large and complex data types

- gold Open Access which fosters wider collaboration and increased citations

- maximum visibility for your research: over $100 \mathrm{M}$ website views per year

At BMC, research is always in progress.

Learn more biomedcentral.com/submissions 